PROCEEDINGS OF THE

AMERICAN MATHEMATICAL SOCIETY

Volume 126, Number 6, June 1998, Pages 1747-1749

S $0002-9939(98) 04214-2$

\title{
A CHARACTERIZATION OF THE HILBERT TRANSFORM
}

\author{
NICOLA ARCOZZI AND LUIGI FONTANA
}

(Communicated by J. Marshall Ash)

\begin{abstract}
In this note the Hilbert transform is characterized in terms of
\end{abstract} function algebras with respect to pointwise multiplication.

Let $H$ be the Hilbert transform on the real line,

$$
H f(x)=\frac{1}{\pi} \mathrm{P} \cdot \mathrm{V} \cdot \int_{-\infty}^{\infty} f(x-y) \frac{\mathrm{d} y}{y}
$$

for $x \in \mathbb{R}$. $H$ extends to a bounded linear operator on $L^{p}(\mathbb{R})$ for $1<p<\infty$.

There are several ways to characterize $H$. For instance, if $F \in H^{p}$, the analytic Hardy space, with $f=\left.\Re F\right|_{\mathbb{R}}, g=\left.\Im F\right|_{\mathbb{R}}$, then $g=H f$. In this context, the Hilbert transform can be extended as the operator that maps the real part $u$ of a function $F=u+i v$ in $H^{p}, 0<p \leq \infty$, to the imaginary part $v$. Notice that $\bigcup_{0<p} H^{p}$ is an algebra with respect to pointwise multiplication. From this we can easily obtain the following equality:

$$
H\left(f^{2}-(H f)^{2}\right)=2 f H f .
$$

In fact, the restriction to $\mathbb{R}$ of $F^{2}$ is

$$
f^{2}-(H f)^{2}+i 2 f H f
$$

which proves $(*)$. Relation $(*)$ was used by Cótlar $[\mathrm{Co}]$ to prove the boundedness of $H$ on $L^{p}$ and by Gokhberg and Krupnik [GK] to find the exact value of $\|H\|_{p}$, when $p=2^{n}$, which is a special case of the later complete result by Pichorides [Pi].

Formula $(*)$ is essentially an "algebra" condition and it is remarkable that it characterizes the Hibert transform. In fact, let $T$ be a bounded linear operator on $L^{2}(\mathbb{R})$ satisfying

(i) $T$ maps real valued functions into real valued functions,

(ii) $T$ commutes with translations,

(iii) $-T^{2}=I$, the identity operator.

Let $\mathcal{A}$ be the space of functions $F=f+i T f$, where $f \in L^{2}(\mathbb{R})$ is real valued. Then, the following is true.

Theorem 1. If $\mathcal{A}$ has the property that $F^{2} \in \mathcal{A}$ whenever $F \in \mathcal{A}$ and $f^{2} \in L^{2}$, then $T= \pm H$ and $\mathcal{A}=H^{2}$.

The proof relies on the following Lemma.

Received by the editors August 20, 1996 and, in revised form, December 1, 1996.

1991 Mathematics Subject Classification. Primary 42A50.

(C)1998 American Mathematical Society 
Lemma 1. Let $E \subset \mathbb{R}$ be a measurable set and $\chi_{E}$ be its characteristic function. Suppose that

(a) $\operatorname{supp}\left(\chi_{E \cap[-R, R]} * \chi_{E \cap[-R, R]}\right) \subseteq E$, for all $R>0$,

(b) $-E=E^{c}$, the complement of $E$.

Then either $\chi_{E}=\chi_{(0, \infty)}$ a.e., or $\chi_{E}=\chi_{(-\infty, 0)}$ a.e..

Proof. Suppose $|E \cap(0, \infty)|>0$. Then there exists $R>0$ such that $|E \cap[0, R]|>0$. Since $\chi_{E \cap[0, R]} * \chi_{E \cap[0, R]}$ is continuous and supported in $[0, \infty)$, (a) implies that $E$ contains an interval $(\alpha, \beta)$ with $0<\alpha<\beta$. By (a) again, $E \supseteq \bigcup_{N=1}^{\infty}(N \alpha, N \beta) \supseteq$ $(\gamma, \infty)$, for some $\gamma>0$. Likewise, if $|E \cap(-\infty, 0)|>0$, we have $E \supset(-\infty, \delta)$ for some $\delta$. By (b), these conditions cannot both hold. Thus, either $E \subset(0, \infty)$ or $E \subset(-\infty, 0)$ modulo a nullset, and then (b) implies the desired result.

Proof of Theorem 1. By properties (ii) and (iii) of $T$, we have that $(\hat{T f})(\xi)=$ $m(\xi) \hat{f}(\xi)$, for all $f \in L^{2}(\mathbb{R})$, where, for some measurable set $E \subset \mathbb{R}, m(\xi)=-i$ if $\xi \in E$ and $m(\xi)=i$ if $\xi \in E^{c}$. (i) implies that $\chi_{E^{c}}=\chi_{-E}$ a.e., and we can assume that $E^{c}=-E$ by modifying $E$ on a nullset.

It is easy to see that $F=f+i g \in \mathcal{A}$ if and only if $\hat{F}(\xi)=0$ for $\xi \in E^{c}$. Let $F$ be such that $\hat{F}=\chi_{E \cap[-R, R]}, R>0$. Then $F \in \mathcal{A} . F^{2} \in L^{2}(\mathbb{R})$ because $\hat{F} * \hat{F}$ is a continuous function with compact support. By the hypothesis, $F^{2} \in \mathcal{A}$, and therefore $\operatorname{supp}\left(\chi_{E \cap[-R, R]} * \chi_{E \cap[-R, R]}\right)=\operatorname{supp}\left(F^{2}\right)^{\wedge} \subseteq E$.

By the Lemma, either $E=(0, \infty)$, or $E=(-\infty, 0)$, modulo a set of measure zero, hence $T=H$ or $T=-H$.

The theorem has analogues if we replace $\mathbb{R}$ with $\mathbb{S}^{1}$ or $\mathbb{Z}$.

Theorem 2. (a) Let $T$ be a bounded linear operator on $L^{2}\left(\mathbb{S}^{1}\right)$ that satisfies (i)(iii) and let $\mathcal{A}$ be the linear space of functions $F=f+i T f$ with $f \in L^{2}\left(\mathbb{S}^{1}\right)$, real valued, such that $\hat{f}(0)=0$. Suppose that $\mathcal{A}$ enjoys the same hypothesis as in Theorem 1. Then $T= \pm H$, where $H$ is now the conjugate function operator.

(b) Let $T$ be a bounded linear operator on $L^{2}(\mathbb{Z})$ that satisfies $(i)$-(iii) and let $\mathcal{A}$ be the linear space of sequences $F=f+i T f$ with $f \in L^{2}(\mathbb{Z})$, real valued. Then $\mathcal{A}$ cannot satisfy the same hypothesis as in Theorem 1. Namely, there exists $F \in \mathcal{A}$ such that $f^{2} \in L^{2}(\mathbb{Z})$, but $F^{2} \notin \mathcal{A}$.

The proof of (a) and (b) follows the same lines as the proof of Theorem 1 . In particular, in case (b) we get a contradiction by requiring that a subset $E$ of $\mathbb{S}^{1}$ satisfy assumptions (a) and (b) of Lemma 1.

There are non translation invariant operators $T$ on $L^{2}(\mathbb{R})$ such that $T$ and the associated space $\mathcal{A}$ satisfy (i), (iii) and the hypothesis of Theorem 1 . It suffices to consider spaces $\mathcal{A}$ of holomorphic functions on suitable domains, $T$ being the conjugate function operator. We do not know whether all the operators satisfying the above properties can be obtained in this way.

We thank Alberto Setti, Enrico Laeng and the referee for their comments and for pointing us to references.

\section{REFERENCES}

[Co] M. Cótlar, A combinatorial inequality and its applications to $L^{2}$ spaces, Revista Mat. Cuyana, 1, 2, 1955, pp. 105-168. MR 18:219a

[Du] P. L. Duren, Theory of $H^{p}$ spaces, Academic Press, New York, London, 1970. MR 42:3552 
[GK] T.S. Gokhberg, N. Ya. Krupnik, Norm of the Hilbert tranformation in the $L^{p}$ space, Func. An. and its Appl, 1968, pp. 180-1.

[Pi] S. K. Pichorides, On the best value of the constants in the theorems of M. Riesz, Zygmund and Kolmogorov, Studia Math., 44, 1972, pp. 165-179. MR 47:702

[SW] E. M. Stein, G. Weiss, Introduction to Fourier analysis on Euclidean spaces, Princeton U.P., Princeton N.J., 1971. MR 46:4102

Università di Milano, Dipartimento di Matematica, "Federico Enriques", Via C. SalDini, 50, 20133 Milano, Italy

E-mail address: arcozzi@mat.unimi.it

E-mail address: fontana@mat.unimi.it 